\title{
A two-channel Fabry-Perot interferometer with thermoelectric-cooled CCD detectors for neutral wind measurement in the upper atmosphere
}

\author{
K. Shiokawa ${ }^{1}$, T. Kadota ${ }^{1}$, Y. Otsuka ${ }^{1}$, T. Ogawa ${ }^{1}$, T. Nakamura ${ }^{2}$, and S. Fukao ${ }^{2}$ \\ ${ }^{1}$ Solar-Terrestrial Environment Laboratory, Nagoya University, Toyokawa 442-8507, Japan \\ ${ }^{2}$ Radio Science Center for Space and Atmosphere, Kyoto University, Gokanosho, \\ Uji, Kyoto 611-0011, Japan
}

(Received March 7, 2003; Revised April 21, 2003; Accepted April 28, 2003)

\begin{abstract}
We describe performance of a new two-channel Fabry-Perot interferometer (FPI) with two thermoelectric-cooled $\mathrm{CCD}$ detectors to measure neutral winds for both the 558-nm (mesopause region) and 630-nm (thermosphere) airglow emissions simultaneously at Shigaraki $\left(34.8^{\circ} \mathrm{N}, 136.1^{\circ} \mathrm{E}\right)$, Japan. The employment of the thermoelectric cooling system enables us continuous automatic operation of the FPI since October 2000. The random errors of wind measurement are estimated to be $\sim 5-8 \mathrm{~m} / \mathrm{s}$ and $\sim 10-50 \mathrm{~m} / \mathrm{s}$ for $558 \mathrm{~nm}$ and $630 \mathrm{~nm}$, respectively. The wind velocities obtained by the FPI $(558$ and $630 \mathrm{~nm})$ fit well with those obtained by the collocated Middle and Upper (MU) atmosphere radar.
\end{abstract}

Key words: Fabry-Perot interferometer, airglow, thermospheric wind, mesospheric wind.

\section{Introduction}

Measurements of atmospheric emissions, such as aurora and airglow, using Fabry-Perot interferometers (FPIs) have enabled the remote sensing of atmospheric wind and temperature from the ground and in space (e.g., Rees et al., 1984; Killeen and Roble, 1988; Hernandez and Roble, 1995; Nakajima et al., 1995; Aruliah et al., 1996; Dyson et al., 1997; Plagmann et al., 1998; Biondi et al., 1999). Some of these FPIs can measure different airglow/auroral lines by changing the interference filter as a pre-disperser. However, such a system does not allow the simultaneous measurement of different lines. Ishii et al., (1997) developed an imaging FPI with two sets of filters and imaging detectors below the etalon to measure OI (558 and $630 \mathrm{~nm}$ ) auroral/airglow lines simultaneously. Biondi et al. (1995) and Shiokawa et al. (2001) have used a new device, highly sensitive liquid-nitrogen cooledCCD detectors, as an imaging detector for their FPI. These authors, however, have pointed out that the use of liquid nitrogen for CCD cooling causes unexpected movement of the CCD detector of 0.1-0.2 pixels, which directly affects the wind velocity determination in the high-resolution interferometry of the FPI. Moreover, unmanned (automated) operation of the FPI measurement is difficult for the liquid nitrogen cooling system.

In this paper, we report performance of a two-channel FPI, which has two sets of filters and cooled-CCD detectors below the etalon with thermoelectric cooling system (without liquid nitrogen), in order to measure OI (558 and $630 \mathrm{~nm})$ airglow lines simultaneously. Automatic operation of the FPI using the thermoelectric cooling system was started beginning in October 2000. The wind velocities measured by the

Copy right(C) The Society of Geomagnetism and Earth, Planetary and Space Sciences (SGEPSS); The Seismological Society of Japan; The Volcanological Society of Japan; The Geodetic Society of Japan; The Japanese Society for Planetary Sciences.
FPI fit well with those obtained from radar for both airglow lines.

\section{Instrumentation}

Figure 1 shows a schematic of the FPI used in the present study. It has telecentric front optics to make a parallel light beam (maximum angle $=0.7^{\circ}$ ) through the etalon (ET, $d=15$ $\mathrm{mm}, \phi=116 \mathrm{~mm})$, and two CCD detectors $(512 \times 512$ pixels) with interference filters to measure the airglow emissions at 558 and $630 \mathrm{~nm}$ separately. The front optics A has a zoom lens with a field-of-view of $9.5^{\circ}$ as an objective and a mirror at the top for azimuthal scanning.

The original system of the present FPI had one fish-eye imaging lens as the front optics and three CCD detectors with a liquid-nitrogen cooling system (Shiokawa et al., 2001). Because the imaging FPI showed significant ambiguity in its measurements of the absolute value of wind velocity, we modified the imaging FPI into an azimuthal-scanning FPI by replacing the front telecentric optics from those with a fisheye lens to those with a zoom lens and a scanning mirror. The CCD detectors with a liquid-nitrogen cooling system were also replaced by those with a thermoelectric cooling system, because the liquid-nitrogen cooling system causes unexpected displacement of the CCD detectors, as described by Shiokawa et al. (2001). One of the CCD detectors used to measure 839.9-nm airglow $(\mathrm{OH})$ was removed from the present system.

The FPI measures the Doppler shift of the airglow emissions at a zenith angle of $50^{\circ} \pm 4.75^{\circ}$ and in the four azimuthal directions of N, S, E, and W. Assuming that the horizontal wind is uniform in the field-of-view of the FPI and that the vertical wind velocity is negligible compared to the horizontal wind velocity, the FPI measures line-of-sight components of horizontal wind vectors by taking a difference in the interference fringe locations in opposite directions $(\mathrm{N}$ - 


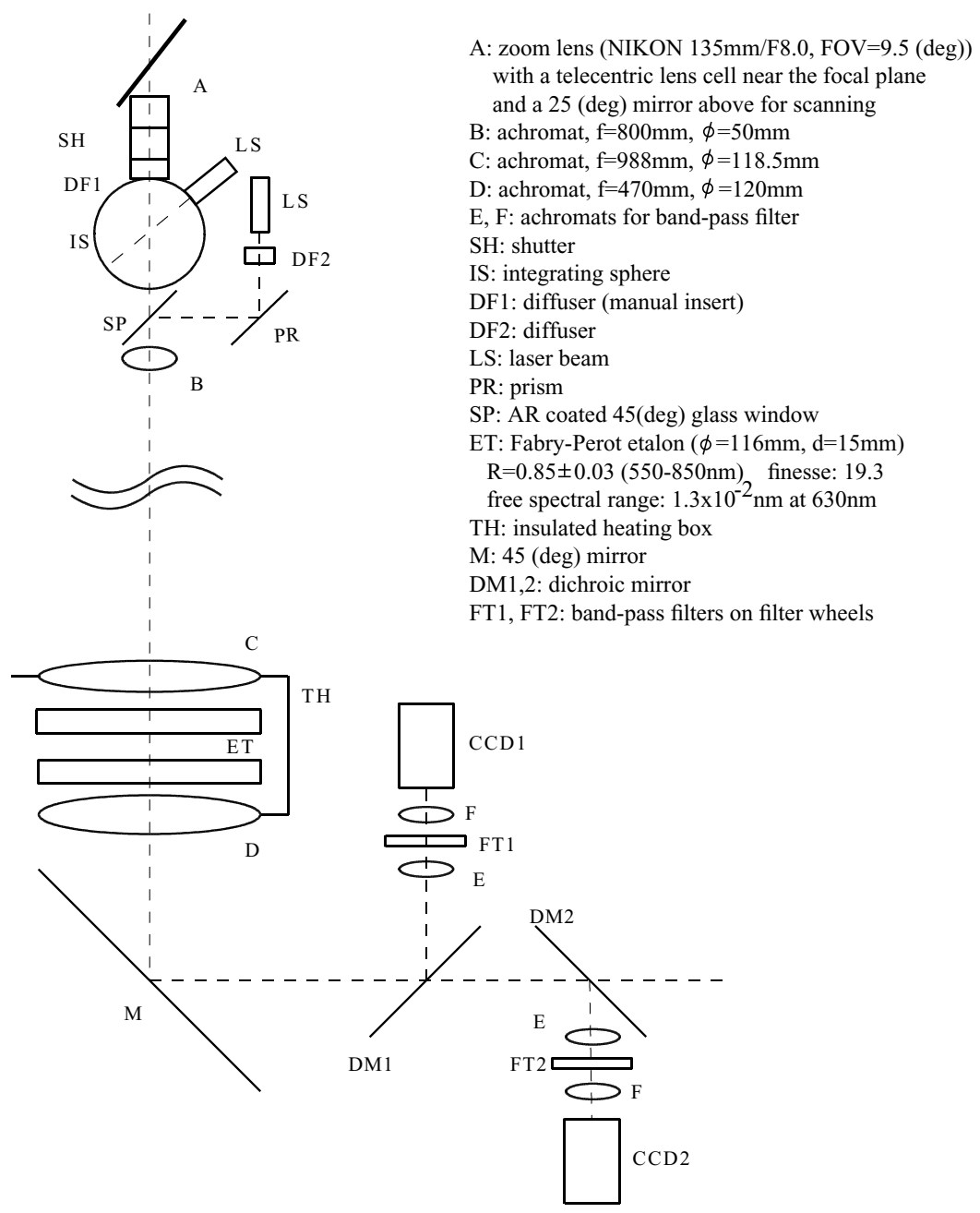

Fig. 1. Schematic diagram of the two-channel Fabry-Perot interferometer.

S and E-W). These assumptions usually hold in the midlatitudes, while they are not applicable for high-latitude auroral zone (Smith, 1998).

Continuous measurement with the FPI has been carried out since October 2000 at Shigaraki $\left(34.8^{\circ} \mathrm{N}, 136.1^{\circ} \mathrm{E}\right)$, Japan, with an exposure time of $13.5 \mathrm{~min}$ for each direction. The time resolution used to obtain a set of zonal and meridional wind velocities is one hour. This time resolution is obtained by $2 \times 2$ binning of the CCD pixels, which makes the output count four times larger than that without the binning. Since October 2001, a higher time resolution of 15 $\min$ (3.5-min exposure for each direction) has been achieved using $4 \times 4$ binning of the CCD pixels.

Figures 2(a) and (b) show examples of airglow fringes obtained at Shigaraki with the FPI for 630- and 558-nm airglow, respectively, at 1948:00-2001:30 LT (13.5-min exposure) on March 26, 2001. The left panels show fringe images in the southward direction. Because of the interference by the etalon in the optics, the line emission from the airglow produces concentric circles (fringes) on the CCD detectors. The right panels show fringes obtained by integrating the images in the left panels for all azimuthal angles of the fringe circles. The relatively high background counts $(\sim 800$ in Fig. 2(a) and $\sim 600$ in Fig. 2(b)) are not because of the background continuum emission from the sky, but mainly because of the read-out offset of the CCD. We will discuss the effect of this CCD read-out noise in Section 4.

By fitting a Gaussian function to the fringes in the right panels, we determine the peak locations of the first and second fringes in units of radius squared, which can be directly converted to wind velocities. For example, northward wind velocity $v_{N}$ can be obtained by the following equation:

$$
v_{N}=\frac{c}{\cos \theta} \frac{r_{S}^{2}-r_{N}^{2}}{4 f^{2}-\left(r_{N}^{2}+r_{S}^{2}\right)}
$$

where $r_{N}$ and $r_{S}$ are the peak location (radius) of the northward and southward fringes, respectively, $f=470 \mathrm{~mm}$ is the focal length of the collimator lens (D in Fig. 1), $c$ is the speed of light, and $\theta$ is the angle of the line-of-sight from the horizontal plane. Equation (1) is derived from the interference condition

$$
\begin{aligned}
m & =\frac{2 \mu d}{\lambda_{0}\left(1+v_{N} \cos \theta / c\right)}\left(1-r_{N}^{2} / 2 f^{2}\right) \\
& =\frac{2 \mu d}{\lambda_{0}\left(1-v_{N} \cos \theta / c\right)}\left(1-r_{S}^{2} / 2 f^{2}\right)
\end{aligned}
$$

where $m$ is the integer number of the interference, $\mu$ is the refractive index, and $\lambda_{0}$ is the wavelength of the airglow without the Doppler shift. Because $4 f^{2}-\left(r_{N}^{2}+r_{S}^{2}\right) \simeq 4 f^{2}$ in 


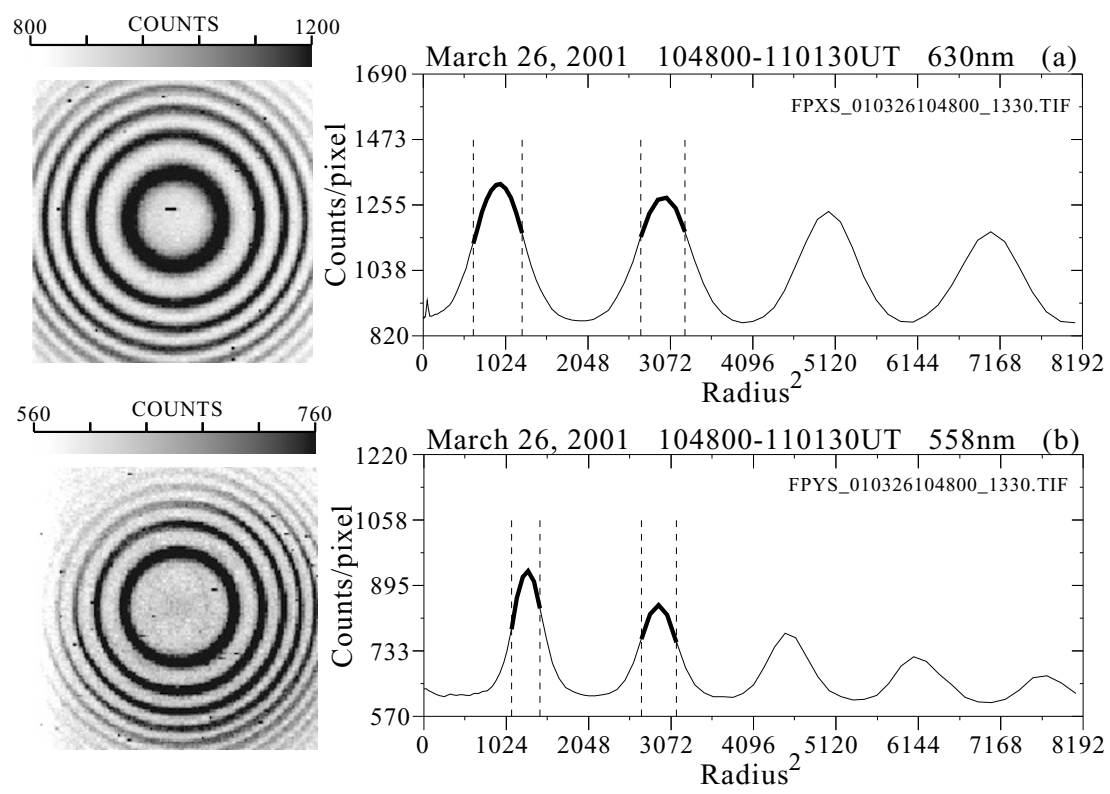

Fig. 2. Airglow fringes obtained by the FPI in the southward direction (zenith angle: $\left.50^{\circ} \pm 4.75^{\circ}\right)$ at 1048:00-1101:30 UT (1948:00-2001:30 LT) on March 26, 2001, at Shigaraki, Japan. Left, fringe images (top (a): 630-nm airglow, bottom (b): 558-nm airglow), and right, cross sections of the fringe images integrated for all azimuthal angles. Black spots in the fringe images are caused by the incidence of cosmic rays onto CCD pixels. The exposure time for obtaining the airglow fringes is $13.5 \mathrm{~min}$. The count rate is indicated as the number of counts per each CCD pixel. The actual count is much higher because of azimuthal integration. The thick solid curves in the right panels indicate the Gaussian functions fitted to the fringes, for the selected region of the first and second fringes shown by the vertical dashed lines.

Eq. (1), the FPI measures the northward wind velocity from the difference between the northward and southward fringe peak locations.

It is interesting to note that for the zero vertical wind velocity, the etalon spacing $d$ can be measured by using the value of $r_{N}^{2}+r_{S}^{2}$, i.e.,

$$
d=\frac{m \lambda_{0}}{\mu} \frac{2 f^{2}}{4 f^{2}-\left(r_{N}^{2}+r_{S}^{2}\right)}
$$

By calculating $r_{N}^{2}+r_{S}^{2}$, we can monitor the etalon spacing drift in the FPI.

\section{Observation}

Figures 3(a)-(g) show the directional wind (relative variation of $r^{2}$ in units of wind velocity), etalon gap drift estimated from $r_{E}^{2}+r_{W}^{2}$ and $r_{N}^{2}+r_{S}^{2}$ in units of wind velocity, fitting error, peak counts, and eastward and northward wind velocities, respectively, obtained for 558-nm airglow at Shigaraki on March 26, 2001. The interference condition (2) was used to convert the unit from radius squared to $\mathrm{m} / \mathrm{s}$. Figures $4(\mathrm{a})-(\mathrm{g})$ show the same parameters measured for $630-\mathrm{nm}$ airglow on the same night. Both the mesospheric (Figs. 3(f) and 3(g)) and thermospheric (Figs 4(f) and 4(g)) wind velocities show nocturnal variations. The variations in Fig. 4(f) and 4(g) represent the typical tidal variations in the nighttime midlatitude thermosphere, i.e., a southward wind around midnight and a changing trend from eastward before midnight to westward after midnight (e.g., Biondi et al., 1999; Kawamura et al., 2000).

To check the reliability of the wind velocities measured with the FPI, we simultaneously observed wind velocities with the FPI and the MU radar in both the mesosphere and the thermosphere at Shigaraki on March 26, 2001, during the MTEC-E (Mesosphere-Thermosphere Experiments for Coupling Studies at Equinoxes) campaign. The MU radar simultaneously measured the mesospheric wind velocities from meteor echoes (Nakamura et al., 1991) and the thermospheric wind velocities from ion drift measurement (Oliver et al., 1998) with a time resolution of 1.5-2 hours.

The wind velocities measured by the MU radar from meteor echoes are plotted in Figs. 3(f) and 3(g) as dashed curves for altitudes of 90, 92, and $94 \mathrm{~km}$ (around the typical altitude of the 558-nm airglow layer). The radar wind velocities at 92 $\mathrm{km}$ are closest to the FPI wind velocities in both the northward and eastward directions, with a maximum difference of $\sim 20 \mathrm{~m} / \mathrm{s}$.

The thermospheric wind velocities estimated by the MU radar from the ion drift measurement are plotted in Fig. 4(g). The MU radar can measure ion drift velocity in the ionospheric $F$-layer along the local magnetic field line. The ion drift velocity is the vector summation of the meridional neutral wind velocity through ion-neutral collision and the fieldaligned ion diffusion velocity. By taking the diffusion velocity from the MSIS-86 model atmosphere, we can convert the measured ion drift velocity into the meridional neutral wind velocity. Thus, the MU radar wind in the thermosphere is not a direct measurement of the neutral motion. On the other hand, the FPI gives the neutral wind velocity directly from the Doppler shift of the 630-nm airglow emission. The comparison in Fig. 4(g) shows a good correspondence between the meridional wind velocities measured by these two techniques, with a maximum difference of $\sim 40 \mathrm{~m} / \mathrm{s}$.

\section{Discussion}

For the wind measurement by FPI, the random error of Gaussian fitting can be estimated from the count rate of the 

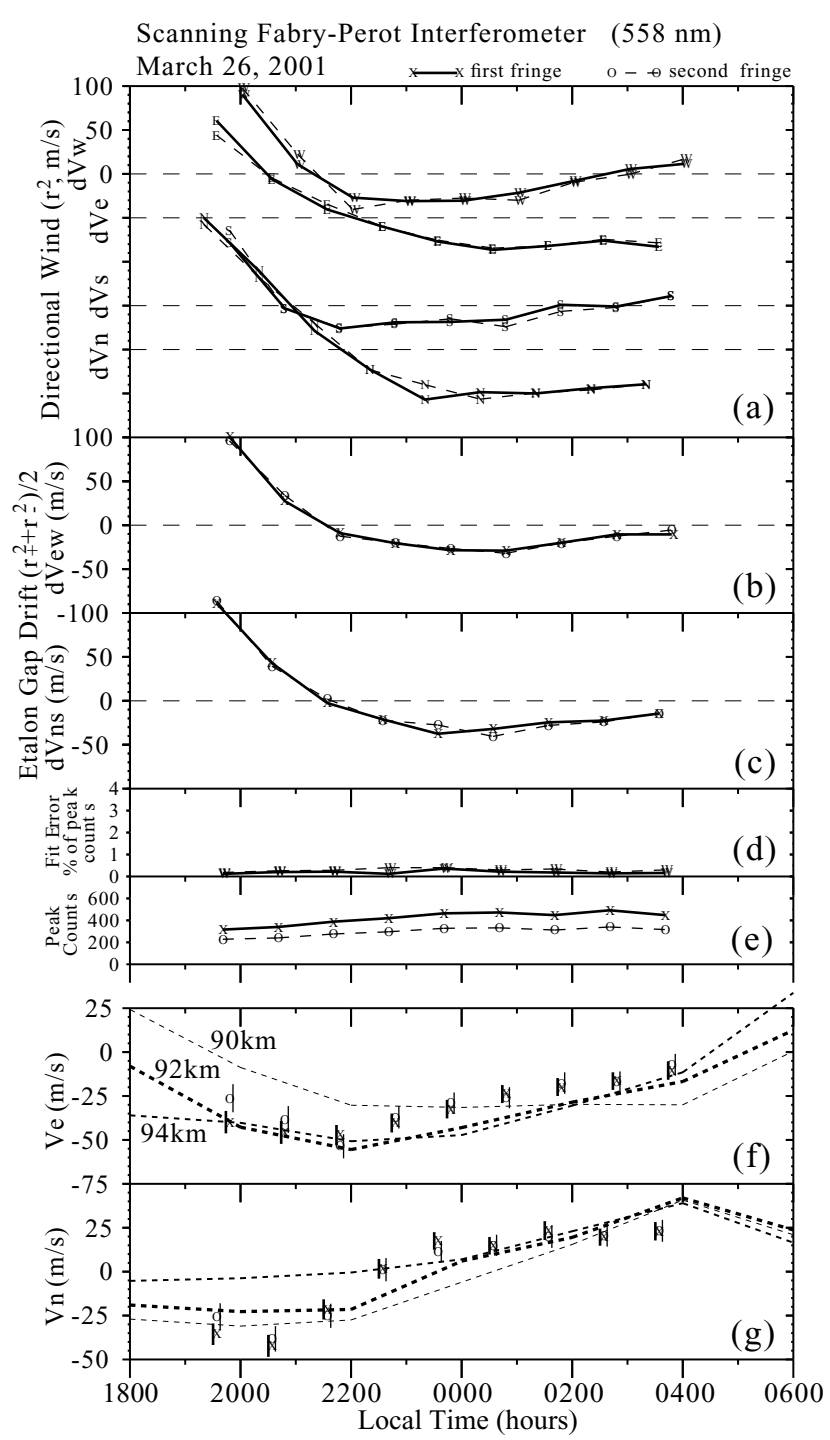

Fig. 3. (a) Directional winds to the north $(d V n)$, south $(d V s)$, east $(d V e)$, and west $(d V w)$ (relative variation of $r^{2}$ in units of wind velocity), (b) and (c) etalon drift estimated from $r_{E}^{2}+r_{W}^{2}$ and $r_{N}^{2}+r_{S}^{2}$, respectively, in units of wind velocity, (d) fitting error (standard deviation of the difference between the fitted Gaussian functions and the observed counts in units of \% from the peak counts; only the maximum of four directions is indicated), (e) peak counts (in units of counts per each CCD pixel; background count is subtracted), and (f) eastward and (g) northward wind velocities, respectively, measured by the FPI for 558-nm airglow at Shigaraki on March 26, 2001. The values for the first and second fringes are indicated by crosses and circles, respectively. The random errors of wind measurement estimated from the Gaussian fitting are indicated by error bars in (f) and (g). The wind velocities measured by the collocated MU radar from meteor echoes are indicated by dashed curves in the bottom two panels for the three altitudes of 90,92 , and $94 \mathrm{~km}$.

fringe data. Figure 2 shows the average counts for each pixel in the fringe images. The peak counts are $\sim 300$ for both 558 $\mathrm{nm}$ and $630 \mathrm{~nm}$. The variations of peak counts on this night are shown in Figs. 3(e) and 4(e). For $558 \mathrm{~nm}$, the counts are $\sim 400$. For $630 \mathrm{~nm}$, they decrease from $\sim 400$ to $\sim 100$ due to the decrease of electron density at night. These counts give root-mean-square (r.m.s.) noise $\sigma_{a}$ of 20 counts and 20-10 counts for $558 \mathrm{~nm}$ and $630 \mathrm{~nm}$, respectively. The read-out noise and dark counts of the CCD give typical r.m.s. noise $\sigma_{\mathrm{CCD}}$ of $\sim 20$, which is comparable to the r.m.s. noise $\sigma_{a}$ of
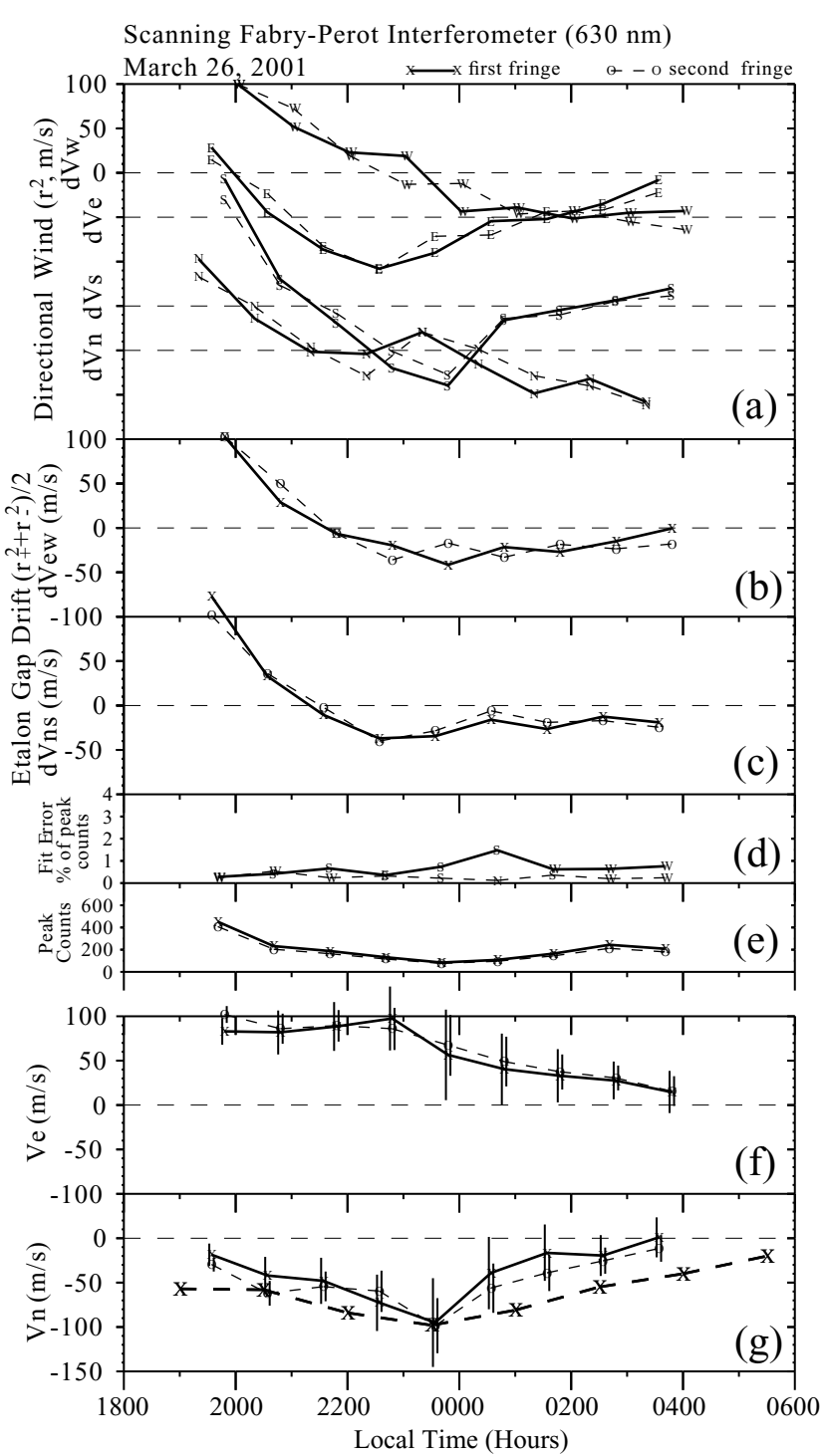

Fig. 4. (a) Directional winds to the north $(d V n)$, south $(d V s)$, east ( $d V e)$, and west $(d V w)$ (relative variation of $r^{2}$ in units of wind velocity), (b) and (c) etalon drift estimated from $r_{E}^{2}+r_{W}^{2}$ and $r_{N}^{2}+r_{S}^{2}$, respectively, in units of wind velocity, (d) fitting error (standard deviation of the difference between the fitted Gaussian functions and the observed counts in units of \% from the peak counts; only the maximum of four directions is indicated), (e) peak counts (in units of counts per each CCD pixel; background count is subtracted), and (f) eastward and (g) northward wind velocities, respectively, measured by the FPI for $630-\mathrm{nm}$ airglow at Shigaraki on March 26, 2001, in the same format as that in Fig. 3. The meridional thermospheric wind velocities estimated from the ion drift measurement by the collocated MU radar are indicated by the thick dashed curve with crosses in the bottom panel $(\mathrm{g})$.

airglow counts. The signal-to-noise ratio $S / N$ is given by (signal counts) / $\left(\sigma_{a}+\sigma_{\mathrm{CCD}}\right)$, which is $\sim 10$ and $\sim 10-3.3$ for $558 \mathrm{~nm}$ and $630 \mathrm{~nm}$, respectively.

These values of $\sigma_{a}, \sigma_{\mathrm{CCD}}$, and $S / N$ are for each CCD pixel. The actual $S / N$ for the fitting is much larger because of the azimuthal integration of the fringes. Typically, 350400 pixels and 650-700 pixels are integrated for the first and second fringes, respectively. Thus, the actual $S / N$ for the Gaussian fitting are $\sqrt{350}-\sqrt{700}$ times larger than the $S / N$ per each pixel. The random errors of Gaussian fitting estimated from these considerations are shown as error bars 
of wind velocities in Figs. 3(f), 3(g), 4(f), and 4(g). The estimated random errors are $\sim 5-8 \mathrm{~m} / \mathrm{s}$ for $558 \mathrm{~nm}$ and $\sim 10$ $50 \mathrm{~m} / \mathrm{s}$ for $630 \mathrm{~nm}$.

It should be noted that the Gaussian fitting to the first and second fringes are made independently. The difference of the wind velocities obtained from the first (crosses) and second (circles) fringes are mostly less than the random errors shown by the error bars.

As indicated by Eq. (3), the etalon spacing drift can be estimated for the present FPI. Figures 3(b), 3(c), 4(b), and 4(c) show a drift in the etalon spacing, particularly for the interval before midnight. This is probably because of the temperature change of the etalon from daytime to nighttime, which was not fully cancelled out by an air conditioner in the FPI hut. After midnight, this spacing drift becomes negligible. Because of this spacing drift, the radius $r$ of the fringes uniformly decreases toward midnight. This effect is seen in Figs. 3(a) and 4(a), which indicate the change of the fringe radii in units of wind velocity. Because the FPI measures the wind velocity from $r_{S}^{2}-r_{N}^{2}$ and $r_{W}^{2}-r_{E}^{2}$, this spacing drift during the time interval between northward and southward exposures directly affects the estimated northward wind velocities. For example, the drift in Fig. 3(b) at 2000 LT was $\sim 70 \mathrm{~m} / \mathrm{s} /$ hour, which causes an offset of $\sim 35 \mathrm{~m} / \mathrm{s}$ (southward) in the final northward wind velocity, because the time difference between the northward and southward exposures was $\sim 30$ min on this night. The exposure sequence was $\mathrm{N}$ $\rightarrow \mathrm{E} \rightarrow \mathrm{S} \rightarrow \mathrm{W}$ on this night, but it was changed to $\mathrm{N} \rightarrow \mathrm{S}$ $\rightarrow \mathrm{E} \rightarrow \mathrm{W}$ starting on April 24, 2001, to minimize the effect of the etalon spacing drift. From October 2001, the exposure time for each direction was reduced from $13.5 \mathrm{~min}$ to 3.5 min. The effect of etalon drift was also reduced by a factor of 4 by this shortening of the exposure time. For example, an etalon drift of $70 \mathrm{~m} / \mathrm{s} /$ hour causes an instrumental offset in the wind velocity of $\sim 8 \mathrm{~m} / \mathrm{s}(=1.17 \mathrm{~m} / \mathrm{s} / \mathrm{min} \times 7 \mathrm{~min}$ (from $\mathrm{N} \rightarrow \mathrm{S})$ ). By monitoring the etalon drift, this instrumental offset can be corrected.

\section{Conclusion}

We have developed a two-channel FPI with two cooledCCD detectors to measure mesospheric and thermospheric wind velocities simultaneously at midlatitudes through the 558- and 630-nm airglow emissions. The FPI was originally developed as an imaging FPI with a fish-eye lens as the front optics and cooled-CCD detectors with liquid-nitrogen cooling system. However, the front imaging optics were replaced by the scanning optics to avoid the inherent ambiguity of the imaging FPI in determining absolute wind velocities. The liquid-nitrogen cooling system was replaced by thermoelectric cooling system to avoid unexpected displacement of the CCD detectors. The employment of the thermoelectric cooling system also enables us automatic operation of the FPI. The random errors of the wind measurement are estimated to be $\sim 5-8 \mathrm{~m} / \mathrm{s}$ and $\sim 10-50 \mathrm{~m} / \mathrm{s}$ for $558 \mathrm{~nm}$ and $630 \mathrm{~nm}$, respectively, for the data of March 26, 2001.

Comparison with the MU radar winds of both the mesosphere and the thermosphere for the data of March 26, 2001, gives reasonable agreement, with a maximum difference of $\sim 20 \mathrm{~m} / \mathrm{s}$ (mesospheric winds for 558-nm airglow) and $\sim 40$ $\mathrm{m} / \mathrm{s}$ (thermospheric winds for 630-nm airglow). The FPI has routinely measured mesospheric and thermospheric wind at Shigaraki since October 2000. Summary plots from these routine observations (similar to Figs. 3 and 4) are available at http: //stab2 . stelab. nagoya-u.ac.jp/omti/.

Acknowledgments. We are grateful to M. Ishii of the Communications Research Laboratory for his helpful comments and suggestions throughout the development of both the hardware and software of the Fabry-Perot interferometer. We also acknowledge S. Okano, M. Taguchi, M. Kubota, T. Sakanoi, K. Sakanoi, and T. Nakajima for their helpful comments. M. Satoh, Y. Katoh, T. Katoh, M. K. Ejiri, C. Ihara, and T. Shimomai of the Solar-Terrestrial Environment Laboratory, Nagoya University, supported the continuous operation of the interferometer and replacement of the front optics. The MU radar at Shigaraki belongs to and is operated by the Radio Science Center for Space and Atmosphere, Kyoto University. This work was supported by Grant-in-Aid of the Ministry of Education, Culture, Sports, Science, and Technology of Japan $(11440145,13573006$, and 13136201).

\section{References}

Aruliah, A. L., A. D. Farmer, D. Rees, U. Brändström, The seasonal behavior of high-latitude thermospheric winds and ion velocities observed over one solar cycle, J. Geophys. Res., 101, 15,701-15,711, 1996.

Biondi, M. A., D. P. Sipler, M. E. Zipf, and J. L. Baumgardner, All-sky Doppler interferometer for thermospheric dynamics studies, Appl. Opt., 34, 1646-1654, 1995.

Biondi, M. A., S. Y. Sazykin, B. G. Fejer, J. W. Meriwether, and C. G. Fesen, Equatorial and low latitude thermospheric winds: Measured quiet time variations with season and solar flux from 1980 to 1990, J. Geophys. Res., 104, 17,091-17,106, 1999.

Dyson, P. L., T. P. Davies, M. L. Parkinson, A. J. Reeves, P. G. Richards, and C. E. Fairchild, Thermospheric neutral winds at southern mid-latitudes A comparison of optical and ionosonde $h_{m} F_{2}$ methods, J. Geophys. Res., 102, 27,189-27,196, 1997.

Hernandez, G. and R. G. Roble, Thermospheric nighttime neutral temperature and winds over Fritz Peak Observatory: Observed and calculated solar cycle variation, J. Geophys. Res., 100, 14,647-14,659, 1995.

Ishii, M., S. Okano, E. Sagawa, S. Watari, H. Mori, I. Iwamoto, and Y. Murayama, Development of Fabry-Perot interferometers for airglow observations, Proc. NIPR Symp. Upper Atmos. Phys., 10, 97-108, 1997.

Kawamura, S., Y. Otsuka, S.-R. Zhang, S. Fukao, and W. L. Oliver, A climatology of middle and upper atmosphere radar observations of thermospheric winds, J. Geophys. Res., 105, 12,777-12,788, 2000.

Killeen, T. L. and R. G. Roble, Thermosphere dynamics: Contributions from the first 5 years of the Dynamics Explorer program, Rev. Geophys., 26, 329-367, 1988.

Nakajima, H., S. Okano, H. Fukunishi, and T. Ono, Observations of thermospheric wind velocities and temperatures by the use of a Fabry-Perot Doppler imaging system at Syowa Station, Antarctica, Appl. Opt., 34, 8382-8395, 1995.

Nakamura, T., T. Tsuda, M. Tsutsumi, K. Kita, T. Uehara, S. Kato, and S. Fukao, Meteor wind observations with the MU radar, Radio Sci., 26, 857-869, 1991.

Oliver, W. L., Y. Otsuka, and S. Fukao, MST radar measurement of ionospheric $F$ region winds: The "layer wind" technique, Radio Sci., 33, 941948, 1998.

Plagmann, M., S. H. Marsh, W. J. Baggaley, R. G. T. Bennett, K. A. Deutsch, G. J. Fraser, G. Hernandez, B. N. Lawrence, G. E. Plank, and R. W. Smith, Annual variation of airglow heights derived from wind measurements, Geophys. Res. Lett., 25, 4457-4460, 1998.

Rees, D., A. H. Greenaway, R. Gordon, I. McWhirter, P. J. Charleton, and A. Steen, The Doppler imaging system: Initial observations of the auroral thermosphere, Planet. Space Sci., 32, 273-285, 1984.

Shiokawa, K., T. Kadota, M. K. Ejiri, Y. Otsuka, Y. Katoh, M. Satoh, and T. Ogawa, Three-channel imaging Fabry-Perot interferometer for midlatitude airglow measurement, Appl. Opt., 40, 4286-4296, 2001.

Smith, R. W., Vertical winds: A tutorial, J. Atmos. Terr. Phys., 60, 14251434, 1998 .

K. Shiokawa (e-mail: shiokawa@stelab.nagoya-u.ac.jp), T. Kadota, Y. Otsuka, T. Ogawa, T. Nakamura, and S. Fukao 